\title{
Frequency, Risk Factors, Prognosis, and Genetic Polymorphism of the Cyclooxygenase-1 Gene for Aspirin Resistance in Elderly Chinese Patients with Cardiovascular Disease
}

\author{
Li Fan $^{a}$ Jian Caob Lin Liu ${ }^{a}$ Xiaoli Lia Guoliang Hu ${ }^{a}$ Yixin $\mathrm{Hu}^{\mathrm{a}}$ Bingpo Zhu ${ }^{\mathrm{b}}$ \\ ${ }^{a}$ Clinical Department of South Building and b First Geriatric Cardiology Division, Chinese PLA General Hospital, \\ Beijing, PR China
}

\section{Key Words}

Aspirin resistance $\cdot$ Frequency $\cdot$ Risk factors $\cdot$ Prognosis $\cdot$

Cyclooxygenase-1 gene $\cdot$ Elderly Chinese patients ·

Cardiovascular disease

\begin{abstract}
Background: Cardiovascular disease (CVD) is an important cause of mortality in elderly patients worldwide. Aspirin resistance has been well reported in CVD. Objective: The frequency, risk factors, prognosis, and genetic polymorphism of the cyclooxygenase-1 (COX-1) gene for aspirin resistance have not been reported in elderly patients with CVD. We therefore undertook this study to evaluate these associations among elderly Chinese patients with CVD. Methods: Four hundred thirty-one elderly Chinese patients with CVD receiving daily aspirin therapy ( $\geq 75 \mathrm{mg}$ ) over 1 month were enrolled. Platelet aggregation was measured by light transmission aggregometry (LTA) and thromboelastography platelet mapping assay (TEG) using arachidonic acid (AA) as a stimulus. The median follow-up was 1.8 years. Results: After the median follow-up, aspirin-resistant patients were at an increased risk of the composite endpoint compared to nonresistant patients by $\mathrm{LTA}_{\mathrm{AA}}+\mathrm{TEG}_{\mathrm{AA}}$ (23.7 vs. $9.2 \%, \mathrm{p}=$
\end{abstract}

0.025). Additionally, Cox proportional hazards regression modeling demonstrated that aspirin resistance and cerebrovascular disease were associated with major adverse longterm outcomes (HR for aspirin resistance $=2.31,95 \% \mathrm{Cl} 1.11$ $4.81, p=0.026)$. The variant $\mathrm{G}$-allele of COX-1 rs1330344 $(-1676 \mathrm{~A} / \mathrm{G})$ significantly increased the risk of aspirin resistance defined by $\mathrm{LTA}_{\mathrm{AA}}+\mathrm{TEG}_{\mathrm{AA}}(\mathrm{OR}=1.82,95 \% \mathrm{Cl} 1.13-$ $2.92, p=0.01)$. Conclusions: Aspirin resistance, evaluated by $\mathrm{LTA}_{\mathrm{AA}}+\mathrm{TEG}_{\mathrm{AA}}$, is associated with an increased risk of adverse clinical events in elderly Chinese patients with CVD. The variant G-allele of COX-1 rs1330344 is significantly associated with aspirin resistance defined by $L_{T A} A A+T E G_{A A}$.

Copyright $\odot 2012$ S. Karger AG, Basel

\section{Introduction}

Cardiovascular disease (CVD) is an important cause of mortality in elderly patients worldwide $[1,2]$. Aspirin is the cornerstone of antiplatelet therapy in patients with CVD and has been reported to reduce the risk of ischemic

Li Fan and Jian Cao are equal first authors.

\section{KARGER}

Fax +4161306 1234

E-Mail karger@karger.ch

www.karger.com (c) 2012 S. Karger AG, Basel

0304-324X/13/0592-0122\$38.00/0

Accessible online at:

www.karger.com/ger
Prof. Li Fan

Clinical Department of South Building

Chinese PLA General Hospital

28 Fuxing Road, Beijing 100853 (PR China)

E-Mail calvin301@163.com 
events by $32 \%$ [3]. However, some studies have suggested that the antiplatelet effect of aspirin may not be uniform in all patients [4,5]. Epidemiological surveys have discovered that $0.4-70.1 \%$ of patients have a poor response to aspirin [6], which is defined as aspirin resistance by different methods.

Recently, several meta-analyses revealed that aspirinresistant patients had a significantly increased risk of death, myocardial infarction, or cerebrovascular events compared to nonresistant patients [7-10]. Possible mechanisms of aspirin resistance include poor compliance or an inadequate dose, increased platelet activity, upregulation of a nonplatelet source of thromboxane production, decreased bioavailability, drug interactions, smoking, hyperlipidemia, hyperglycemia, and genetic variability [11-15]. Despite ongoing research, there is currently no clear consensus on the definition and treatment of aspirin resistance.

Recently, we published our observations demonstrating that aspirin resistance or semiresponders, defined by light transmission aggregometry (LTA), are associated with an increased risk of adverse clinical events in elderly male patients with CVD [16]. However, there was no investigation of the association between aspirin resistance and polymorphisms of the Cyclooxygenase-1 $(C O X-1)$ gene. In the present study, we explored the relationship between aspirin resistance and polymorphisms of $C O X-1$ and evaluated other associations with aspirin resistance among elderly Chinese patients with CVD.

\section{Materials and Methods}

\section{Ethical Approval of the Study Protocol}

This study complied with the Declaration of Helsinki. It was approved by the Scientific and Ethics Review Board of the Chinese PLA General Hospital (Beijing, PR China). All patients provided written informed consent prior to inclusion in the study.

\section{Participants}

Initially, we prospectively enrolled 479 patients from April 2008 to June 2010. Patients were recruited from the Wangshoulu area of Beijing. Patients were aged $\geq 65$ years and were being treated for coronary heart disease, hypertension, peripheral arterial disease, or stroke; all patients were on regular treatment with aspirin (75-100 mg daily over 1 month). Patients' use of aspirin was determined by patient interview both at study enrolment and at follow-up. The exclusion criteria included: use of clopidogrel, ticlopidine, dipyridamole or other nonsteroidal anti-inflammatory drugs, or heparin or low-molecular-weight heparin; a major surgical procedure within 1 week of study enrolment; a family or personal history of bleeding disorders; platelet count $<150 \times$ $10^{3} / \mu \mathrm{l}$ or $>450 \times 10^{3} / \mathrm{\mu l}$; hemoglobin $<8 \mathrm{~g} / \mathrm{dl}$; a history of myelo- proliferative disorders, or a history of drug-induced thrombocytopenia. After enrollment, patients with poor compliance $(n=22)$, an inadequate dose $(\leq 75 \mathrm{mg})(\mathrm{n}=15)$, and a history of taking other antiplatelet medications $(\mathrm{n}=11)$ were excluded. Thus, 431 patients were eventually included in the present study.

\section{Blood Sampling}

Blood samples were collected from the antecubital vein into Vacutainer tubes 2-12 $\mathrm{h}$ after the administration of the last dose of aspirin. The first $2 \mathrm{ml}$ of blood drawn by venipuncture was discarded. Four tubes of whole blood, anticoagulated with 3.2\% sodium citrate and lithium heparin, were used for LTA and thromboelastography (TEG). One tube which contained lithium heparin was also used for TEG. In addition, one tube of blood anticoagulated with CTAD (a mixture of citrate, theophylline, adenosine, and dipyridamole) was used for measurements of CD62P (P-selectin) and PAC-1 (activated GP IIb/IIIa receptors). The tubes were filled to capacity and gently inverted $3-5$ times to ensure complete mixture of the anticoagulant. Moreover, four conventional tubes were collected to determine: high-sensitivity $\mathrm{C}$-reactive protein, type-B natriuretic peptide, and protein $\mathrm{C}$; the percentage activity of antithrombin III; routine measurements of blood components and blood lipids, and other biochemical measurements. All assays were processed within $2 \mathrm{~h}$ of blood sampling.

\section{Light Transmission Aggregometry}

Blood samples were centrifuged at $800 \mathrm{rpm}$ for $5 \mathrm{~min}$ to obtain native platelet-rich plasma and further centrifuged at $4,000 \mathrm{rpm}$ for $8 \mathrm{~min}$ to obtain platelet-poor plasma. The platelet count of the platelet-rich plasma was assessed and adjusted to between $200 \times$ $10^{3} / \mu \mathrm{l}$ and $300 \times 10^{3} / \mu \mathrm{l}$ using platelet-poor plasma. Aggregation was performed using arachidonic acid (AA; $0.5 \mathrm{mM}$ ) with a Chrono-Log aggregometer (Chrono-Log, Havertown, Pa., USA).

\section{TEG Platelet Mapping Assay}

The TEG platelet mapping assay (Hemoscope, Niles, Ill., USA) relies on the measurement of platelet function through clot strength. AA $(1 \mathrm{mmol} / \mathrm{l})$ was added to activator $\mathrm{F}$ to measure the degree of thromboxane $\mathrm{A}_{2}\left(\mathrm{TXA}_{2}\right)$-induced platelet aggregation. This methodology is described elsewhere [17].

\section{Other Laboratory Analyses}

The hemoglobin assay and platelet counts were performed on a SYSMEX- XE2100 analyzer (SYSMEX, Kobe, Japan). Serum creatinine, blood lipid, homocysteine, high-sensitivity C-reactive protein, type-B natriuretic peptide and uric acid, and other basic biochemical blood tests were measured by standard chemical and enzymatic commercial methods in a cobas 6000 analyzer series (Roche Diagnostics). The percentage activity of antithrombin III and protein $\mathrm{C}$ was tested by a STA-R Evolution coagulation analyzer (Stago, Paris, France).

\section{Single-Nucleotide Polymorphism Detection}

DNA samples were extracted from peripheral blood obtained from all patients by a standard phenol-chloroform method. Single-nucleotide polymorphism (SNP) detection was performed using the Sequenom MassARRAY iPLEX platform (Sequenom, USA). PCR primers and single base extension probes were designed using Assay Design 3.1 software from Sequenom. SNPs 
were genotyped by the Sequenom MassArray system according to the manufacturer's instructions. PCR was performed using iPlex chemistry according to the recommendations of the manufacturer as found in the MassArray iPlex standard operating procedure. Data analysis was performed using MassArray Typer software version 4.0 .

\section{Definition of Aspirin Resistance}

The definition of aspirin resistance was $\geq 20 \%$ AA aggregation according to LTA [18]. Using TEG, aspirin resistance was defined as $\geq 50 \%$ aggregation induced by AA [17]. Additionally, we used the combination of LTA ( $\geq 20 \%$ AA-aggregation) and TEG ( $\geq 50 \%$ AA aggregation) criteria to define aspirin resistance.

\section{Study Endpoints}

The primary end point was the composite of adverse clinical events, including death, myocardial infarction, stroke, transient ischemic attack, and unstable angina. Death was defined as death from vascular and nonvascular causes. Myocardial infarction was defined by the European Society of Cardiology/American College of Cardiology criteria [19]. Stroke was defined as an acute neurologic vascular event lasting more than $24 \mathrm{~h}$. Transient ischemic attack was defined as an acute neurologic vascular event lasting less than $24 \mathrm{~h}$. Unstable angina was defined according to the American College of Cardiology Foundation/American Heart Association criteria [20].

Follow-Up

The follow-up was completed in all patients by telephone interviews and review of medical records. Personnel performing data collection were blind to aspirin responsiveness. The followup time ranged from 1 to 3 years. The median follow-up time was 1.8 years.

\section{Statistical Analyses}

Continuous variables are expressed as means \pm SD. Univariate tests or Kruskal-Wallis tests (if the distribution was not normal) were used to compare the continuous variables among the three groups defined by aggregation. For continuous variables, differences between the two groups were evaluated using Student's t test or Mann-Whitney U two-sample tests (if the distribution was not normal). Categorical data and proportions were analyzed using Pearson's $\chi^{2}$ test or, in the case of small expected cell frequencies, exact tests. All SNPs evaluated in this study were tested for deviation from Hardy-Weinberg equilibrium with a $\chi^{2}$ test. The association between COX-1 polymorphisms and aspirin resistance was analyzed in logistic regression models after adjustment for age and gender by SNP status in web and the pairwise $\mathrm{D}^{\prime}$ and $r^{2}$ values were analyzed for linkage disequilibrium. Haploview 4.2 software was used to generate the maximum likelihood estimates of haplotype frequencies. OR and 95\% CI were used to estimate associations of alleles and genotypes between cases and controls. $\mathrm{p}<0.05$ was considered statistically significant. Cumulative survival curves for both aspirin-resistant and nonresistant groups were constructed using the Kaplan-Meier method. Parameters significantly related to the presence of aspirin resistance were determined using binary logistic regression analyses. A Cox proportional hazards regression model was generated to describe risk factors for clinical events (SPSS, Windows, version 14.0; Chicago, Ill., USA).

\section{Results}

\section{Patient Characteristics}

Demographics, as well as a comparison of aspirinresistant patients and nonresistant patients by $\mathrm{LTA}_{\mathrm{AA}}$, $\mathrm{TEG}_{\mathrm{AA}}$, and $\mathrm{LTA}_{\mathrm{AA}}+\mathrm{TEG}_{\mathrm{AA}}$, are provided in table 1 . There were no differences between aspirin-resistant patients and nonresistant patients regarding sex, age, BMI and cardiovascular risk factors, and other biochemical measurements by $\mathrm{LTA}_{\mathrm{AA}}$.

According to $\mathrm{TEG}_{\mathrm{AA}}$, there were no significant differences between the aspirin-resistant and nonresistant groups when comparing: age; being a current smoker; having hypertension, coronary artery disease, cerebrovascular disease, or peripheral arterial occlusive disease, and the baseline platelet count. Aspirin-resistant patients had higher levels of fasting serum glucose, CD62P, and LDL cholesterol compared to nonresistant patients ( $\mathrm{p}=$ $0.006, p=0.003$, and $p=0.047$, respectively). Patients with aspirin resistance were less likely to take nitrate therapy than were nonresistant patients $(\mathrm{p}=0.018)$.

Aspirin resistance and aspirin nonresistance as measured by $\mathrm{LTA}_{\mathrm{AA}}+\mathrm{TEG}_{\mathrm{AA}}$ were not associated with significant differences with regard to: age, being a current smoker, having hypertension, coronary artery disease, or peripheral arterial occlusive disease, and the baseline platelet count. The number of patients with cerebrovascular disease was significantly higher in aspirin-resistant patients compared to nonresistant patients $(\mathrm{p}=0.035)$.

\section{Testing of Platelet Aggregation}

According to $\mathrm{LTA}_{\mathrm{AA}}, 59$ (13.7\%) elderly patients were found to be resistant to aspirin therapy. According to $\mathrm{TEG}_{\mathrm{AA}}, 102$ patients (23.7\%) were aspirin resistant. Of the 102 patients who were aspirin-resistant by TEG $_{\mathrm{AA}}, 38 \mathrm{pa}-$ tients were aspirin resistant by LTA $_{\mathrm{AA}}$. The kappa statistic between these two methods was 0.361. According to $\mathrm{LTA}_{\mathrm{AA}}+\mathrm{TEG}_{\mathrm{AA}}, 38$ (8.8\%) patients were aspirin resistant. The kappa statistic between the two former methods and the combined methods was 0.757 .

\section{Multiple Logistic Regression Analysis}

We did not find significant risk factors using LTA $_{A A}$. $\mathrm{TEG}_{\mathrm{AA}}$ revealed that CD62P levels $(\mathrm{OR}=1.01,95 \% \mathrm{CI}$ $1.00-1.02, \mathrm{p}=0.028)$, fasting serum glucose levels $(\mathrm{OR}=$ $1.32,95 \%$ CI 1.09-1.59, $\mathrm{p}=0.003)$, and administration of nitrates $(\mathrm{OR}=0.44,95 \% \mathrm{CI} 0.23-0.85, \mathrm{p}=0.016)$ were significantly associated with aspirin resistance (table 2). Using $\mathrm{LTA}_{\mathrm{AA}}+\mathrm{TEG}_{\mathrm{AA}}$, cerebrovascular disease $(\mathrm{OR}=$ $1.67,95 \%$ CI 1.17-2.39, $\mathrm{p}=0.005$ ) was a risk factor for as- 
Table 1. Patient demographics by different methods

\begin{tabular}{|c|c|c|c|c|c|c|c|c|c|}
\hline & $\begin{array}{l}\mathrm{AR} \\
\left(\mathrm{LTA}_{\mathrm{AA}}\right) \\
(\mathrm{n}=59)\end{array}$ & $\begin{array}{l}\text { Non-AR } \\
\left(\mathrm{LTA}_{\mathrm{AA}}\right) \\
(\mathrm{n}=372)\end{array}$ & $\begin{array}{l}\mathrm{p} \\
\text { value }\end{array}$ & $\begin{array}{l}\mathrm{AR} \\
\left(\mathrm{TEG} \mathrm{AA}_{\mathrm{AA}}\right) \\
(\mathrm{n}=102)\end{array}$ & $\begin{array}{l}\text { Non-AR } \\
\left(T_{E G} G_{A A}\right) \\
(n=329)\end{array}$ & $\begin{array}{l}\mathrm{p} \\
\text { value }\end{array}$ & $\begin{array}{l}\mathrm{AR} \\
\left(\mathrm{LTA}_{\mathrm{AA}}+\mathrm{TEG}_{\mathrm{AA}}\right) \\
(\mathrm{n}=38)\end{array}$ & $\begin{array}{l}\text { Non-AR } \\
\left(\mathrm{LTA}_{\mathrm{AA}}+\mathrm{TEG}_{\mathrm{AA}}\right) \\
(\mathrm{n}=393)\end{array}$ & $\begin{array}{l}\mathrm{p} \\
\text { value }\end{array}$ \\
\hline Age, years & $74.88 \pm 9.01$ & $73.98 \pm 7.92$ & 0.428 & $74.21 \pm 8.21$ & $74.08 \pm 8.04$ & 0.888 & $75.37 \pm 9.92$ & $74.98 \pm 7.87$ & 0.314 \\
\hline Female, n (\%) & $19(32.2)$ & $129(34.7)$ & 0.710 & $43(42.2)$ & $105(31.9)$ & 0.057 & $11(28.9)$ & $137(34.9)$ & 0.464 \\
\hline Hypertension, n (\%) & $46(77.9)$ & $255(68.5)$ & 0.143 & $74(72.5)$ & $227(69.0)$ & 0.495 & $28(73.7)$ & $273(69.5)$ & 0.588 \\
\hline Coronary artery disease, $\mathrm{n}(\%)$ & $38(64.4)$ & $205(58.1)$ & 0.181 & $60(58.8)$ & $183(55.6)$ & 0.569 & $23(60.5)$ & $220(60)$ & 0.589 \\
\hline PAOD, n (\%) & $5(8.5)$ & $35(9.4)$ & 0.524 & $12(11.8)$ & $28(8.5)$ & 0.322 & $5(13.2)$ & $35(8.9)$ & 0.388 \\
\hline BMI & $25.35 \pm 3.44$ & $25.15 \pm 3.56$ & 0.691 & $25.10 \pm 3.39$ & $25.13 \pm 3.81$ & 0.943 & $24.95 \pm 3.81$ & $25.14 \pm 3.71$ & 0.765 \\
\hline Homocysteine, $\mu \mathrm{mol} / \mathrm{l}$ & $17.36 \pm 6.71$ & $17.17 \pm 8.66$ & 0.883 & $17.24 \pm 7.53$ & $17.19 \pm 8.67$ & 0.957 & $17.19 \pm 5.04$ & $17.20 \pm 8.65$ & 0.997 \\
\hline $\mathrm{BNP}, \mathrm{pg} / \mathrm{ml}$ & $166.74 \pm 214.02$ & $124.38 \pm 220.96$ & 0.205 & $128.60 \pm 189.57$ & $130.25 \pm 229.29$ & 0.950 & $128.73 \pm 162.63$ & $129.95 \pm 224.76$ & 0.976 \\
\hline Hs-CRP, mg/dl & $0.29 \pm 0.26$ & $0.47 \pm 1.15$ & 0.284 & $0.54 \pm 1.49$ & $0.42 \pm 0.92$ & 0.355 & $0.34 \pm 20.30$ & $0.45 \pm 1.12$ & 0.576 \\
\hline CD62P, \% & $17.29 \pm 21.05$ & $15.74 \pm 20.86$ & 0.600 & $21.20 \pm 21.16$ & $14.26 \pm 19.42$ & 0.003 & $19.46 \pm 23.24$ & $15.61 \pm 20.62$ & 0.278 \\
\hline Total cholesterol, $\mathrm{mmol} / \mathrm{l}$ & $4.80 \pm 1.04$ & $5.04 \pm 2.53$ & 0.484 & $5.16 \pm 1.24$ & $4.95 \pm 2.64$ & 0.438 & $4.86 \pm 0.98$ & $5.02 \pm 2.48$ & 0.689 \\
\hline Triglyceride, mmol/l & $1.74 \pm 0.82$ & $1.58 \pm 0.71$ & 0.128 & $1.71 \pm 0.83$ & $1.58 \pm 0.69$ & 0.130 & $1.76 \pm 0.93$ & $1.59 \pm 0.71$ & 0.169 \\
\hline HDL cholesterol, mmol/l & $1.28 \pm 0.36$ & $1.31 \pm 0.36$ & 0.495 & $1.30 \pm 0.36$ & $1.31 \pm 0.36$ & 0.841 & $1.28 \pm 0.37$ & $1.31 \pm 0.36$ & 0.664 \\
\hline LDL cholesterol, mmol/l & $2.81 \pm 0.84$ & $2.88 \pm 0.86$ & 0.581 & $3.02 \pm 1.04$ & $2.82 \pm 0.79$ & 0.047 & $2.85 \pm 0.85$ & $2.87 \pm 0.86$ & 0.857 \\
\hline Uric acid, $\mu \mathrm{mol} / \mathrm{l}$ & $325.71 \pm 96.47$ & $325.65 \pm 86.42$ & 0.997 & $328.87 \pm 85.15$ & $325.59 \pm 88.43$ & 0.981 & $357.09 \pm 77.51$ & $323.40 \pm 87.88$ & 0.075 \\
\hline Platelet count, $\times 10^{3} / \mu \mathrm{l}$ & $217.57 \pm 77.62$ & $206.87 \pm 55.19$ & 0.206 & $215.00 \pm 67.57$ & $206.32 \pm 55.80$ & 0.206 & $220.00 \pm 79.82$ & $207.25 \pm 56.43$ & 0.220 \\
\hline \multicolumn{10}{|l|}{ Medications taken } \\
\hline Statins, n (\%) & $18(30.5)$ & $94(25.3)$ & 0.394 & & & 0.367 & & & 0.226 \\
\hline Bs, n (\%) & & & & & & & & & 0.684 \\
\hline & & & 0.290 & & & 0.018 & & & 0.314 \\
\hline CCBs, $\mathrm{n}(\%)$ & $25(42.4)$ & $132(35.5)$ & 0.307 & $36(35.3)$ & $121(36.7)$ & 0.786 & $12(31.6)$ & $145(36.9)$ & 0.537 \\
\hline \multicolumn{10}{|l|}{ Daily aspirin dose, $\mathrm{n}(\%)$} \\
\hline
\end{tabular}

$\mathrm{AR}=$ Aspirin resistance; non- $\mathrm{AR}=$ non-aspirin resistance; $\mathrm{PAOD}=$ peripheral arterial occlusive disease; $\mathrm{Hs}$ - $\mathrm{CRP}=$ high-sensitivity $\mathrm{C}$-reactive protein; $\mathrm{BNP}=$ type- $\mathrm{B}$ natriuretic peptide; $\mathrm{ACEIs}=$ angiotensin- converting enzyme inhibitors; $\mathrm{ARBs}=$ angiotensin receptor blockers; $\mathrm{CCBs}=\mathrm{calcium}$ channel blockers.

pirin resistance (table 2). Additionally, Cox proportional hazards regression modeling demonstrated that aspirin resistance $(\mathrm{HR}=2.31,95 \% \mathrm{CI} 1.11-4.81, \mathrm{p}=0.026)$ and cerebrovascular disease $(\mathrm{HR}=1.71,95 \%$ CI $1.38-2.12$, $\mathrm{p}<0.001)$ were associated with major adverse long-term outcomes by $\mathrm{LTA}_{\mathrm{AA}}+\mathrm{TEG}_{\mathrm{AA}}$ (table 2$)$.

\section{Follow-Up, Clinical Vascular Events}

During the follow-up period, the frequency of cardiovascular-related events was high in aspirin-resistant patients compared with nonresistant patients by LTA $_{\mathrm{AA}}$ (16.9 vs. 9.4\%). By TEG $\mathrm{AA}_{\mathrm{A}}$, aspirin-resistant patients had more cardiovascular events compared with nonresistant patients (12.7 vs. 9.7\%). The frequency of cardiovascularrelated events was higher in patients with aspirin resis- tance as compared to nonresistant patients by $\mathrm{LTA}_{\mathrm{AA}}+$ TEG $_{\text {AA }}(23.7$ vs. $9.2 \%, p=0.025)$ (table 3). The KaplanMeier curves of the probability of survival without clinical events reached a significant difference between the two groups by $\mathrm{LTA}_{\mathrm{AA}}+\mathrm{TEG}_{\mathrm{AA}}(\mathrm{p}<0.001)$ (fig. 1).

\section{Genetic Association with Aspirin Resistance}

The correlation of aspirin resistance with 6 SNPs, including rs1888943 (-8759C/T), rs1330344 (-1676A/G), rs3842787 (exon 2, 50C/T), rs5787 (exon 4, 323G/A), rs5789 (exon 7, 709C/A), and rs5794 (exon 10, 1330G/A), in COX-1 genes was investigated. rs1888943, rs3842787, rs5787, rs5789, and rs5794 variants were rare in the present study and did not reach statistical significance between aspirin-resistant patients and nonresistant patients 
by the different modes. The variant G-allele of COX-1 rs1330344 (-1676 A/G) showed an increased risk tendency for aspirin resistance defined by $\mathrm{LTA}_{\mathrm{AA}}$ or TEG $\mathrm{AA}$ $(\mathrm{p}=0.06)$ (table 4). However, the variant G-allele of rs1330344 significantly increased the risk of aspirin-resistant patients compared with nonresistant patients $(\mathrm{p}=$ 0.01 ) by $\mathrm{LTA}_{\mathrm{AA}}+\mathrm{TEG}_{\mathrm{AA}}$ (table 4 ).

\section{Discussion}

Among patients with CVD, the present study showed, for the first time, that the frequency of aspirin resistance by $\mathrm{LTA}_{\mathrm{AA}}+\mathrm{TEG}_{\mathrm{AA}}$ was $8.8 \%$ in elderly patients. However, the frequency of aspirin resistance evaluated by $\mathrm{LTA}_{\mathrm{AA}}$ or TEG $\mathrm{AA}_{\text {was }}$ reported as 13.7 and $23.7 \%$, respectively, which was high compared with the combined methods. Because of the lack of a standardized approach to diagnosis, a different frequency of aspirin resistance has been observed in previous studies when using different methods for the detection of aspirin resistance.

Some studies showed that the frequency of aspirin resistance by LTA ranged from 4 to $60 \%[11,18]$. The frequency of aspirin resistance measured by TEG was reported to be from $<1$ to $33 \%$ [21-23]. The frequency of aspirin resistance assessed by PFA-100 was $9.5-46.8 \%$ $[24,25]$. On the other hand, several studies revealed that aspirin resistance may be overestimated using methods that do not use AA as a stimulus [16, 20]. We therefore used the combined methods to evaluate the frequency of aspirin resistance. Interestingly, the frequency of cardiovascular-related events was high in aspirin-resistant patients by $\mathrm{LTA}_{\mathrm{AA}}+\mathrm{TEG}_{\mathrm{AA}}$ compared with aspirin-resistant patients by $\mathrm{LTA}_{\mathrm{AA}}$ or TEG $\mathrm{TAA}_{\mathrm{A}}$. However, the present study demonstrated that aspirin-resistant patients defined by $\mathrm{LTA}_{\mathrm{AA}}+\mathrm{TEG}_{\mathrm{AA}}$ had a significantly increased risk of clinical events. Furthermore, when aspirin resistance was defined by a single method, $\mathrm{LTA}_{\mathrm{AA}}$ or TEG $\mathrm{AA}_{\mathrm{A}}$, aspirin resistance showed an association with COX-1 variants. However, aspirin resistance defined by $\mathrm{LTA}_{\mathrm{AA}}+$ TEG $_{\mathrm{AA}}$ was significantly associated with COX-1 variants. These results indicate that aspirin resistance may not be overestimated by the combined methods of $\mathrm{LTA}_{\mathrm{AA}}+$ TEG $_{\mathrm{AA}}$.

Using multivariate logistic regression analysis, we demonstrated that cerebrovascular disease, fasting serum glucose levels, CD62P levels, and administration of nitrates was associated with aspirin resistance in elderly patients. The number of patients with cerebrovascular disease was significantly higher in those with aspirin re-
Table 2. Results of multiple logistic regression and Cox proportional hazards regression analysis by different methods

a OR for aspirin resistance by TEG $_{\mathrm{AA}}$

\begin{tabular}{|c|c|c|}
\hline & $\begin{array}{l}\text { OR } \\
(95 \% \mathrm{CI})\end{array}$ & $\begin{array}{l}\mathrm{p} \\
\text { value }\end{array}$ \\
\hline \multicolumn{3}{|l|}{ Univariate analysis } \\
\hline Sex & $1.55(0.98-2.45)$ & 0.058 \\
\hline CD62P levels & $1.01(1.00-1.02)$ & 0.004 \\
\hline Fasting serum glucose levels & $1.22(1.05-1.41)$ & 0.009 \\
\hline LDL cholesterol levels & $1.30(1.00-1.68)$ & 0.048 \\
\hline Nitrates therapy & $0.43(0.23-0.81)$ & 0.009 \\
\hline \multicolumn{3}{|l|}{ Multivariate analysis } \\
\hline CD62P levels & $1.01(1.00-1.02)$ & 0.028 \\
\hline Fasting serum glucose levels & $1.32(1.09-1.59)$ & 0.003 \\
\hline Nitrates therapy & $0.44(0.23-0.85)$ & 0.016 \\
\hline \multicolumn{3}{|c|}{ b OR for aspirin resistance by $\mathrm{LTA}_{\mathrm{AA}}+\mathrm{TEG}_{\mathrm{AA}}$} \\
\hline & $\begin{array}{l}\text { OR } \\
(95 \% \text { CI })\end{array}$ & $\begin{array}{l}\mathrm{p} \\
\text { value }\end{array}$ \\
\hline \multicolumn{3}{|l|}{ Univariate analysis } \\
\hline Cerebrovascular disease & $1.44(1.09-1.91)$ & 0.010 \\
\hline Uric acid & $1.01(1.00-1.02)$ & 0.072 \\
\hline \multicolumn{3}{|l|}{ Multivariate analysis } \\
\hline Cerebrovascular disease & $1.67(1.17-2.39)$ & 0.005 \\
\hline
\end{tabular}

c $\mathrm{HR}$ of the composite endpoint according to $\mathrm{LTA}_{\mathrm{AA}}+\mathrm{TEG}_{\mathrm{AA}}$ by Cox proportional hazards regression

\begin{tabular}{llr}
\hline & $\begin{array}{l}\text { OR } \\
(95 \% \mathrm{CI})\end{array}$ & \multicolumn{1}{c}{$\begin{array}{l}\mathrm{p} \\
\text { value }\end{array}$} \\
\hline $\begin{array}{llr}\text { Univariate analysis } \\
\quad \text { Diabetes }\end{array}$ & $1.27(1.01-1.59)$ & 0.034 \\
$\quad$ Aspirin resistance & $2.44(1.36-4.39)$ & 0.003 \\
$\quad$ Cerebrovascular disease & $1.72(1.39-2.11)$ & $<0.001$ \\
Multivariate analysis & & \\
$\quad$ Aspirin resistance & $2.31(1.11-4.81)$ & 0.026 \\
$\quad$ Cerebrovascular disease & $1.71(1.38-2.12)$ & $<0.001$ \\
\hline
\end{tabular}

sistance than in nonresistant patients in the present study. Previous studies showed that the frequency of aspirin resistance was not low in a stroke population (12-56\%) [2628]. Furthermore, Englyst et al. [29] demonstrated that aspirin resistance was associated with stroke severity. Unfortunately, one study demonstrated that clinical events (including myocardial infarction, vascular death, and stroke) occurred in $40 \%$ of aspirin-resistant patients compared with $4.4 \%$ of nonresistant patients ( $\mathrm{p}<0.001$ ), which enrolled 180 post-ischemic stroke patients and followed them over 2 years [30]. The present study showed 
Table 3. Occurrence of composite endpoints in aspirin-resistant and nonresistant patients

\begin{tabular}{|c|c|c|c|c|c|c|c|c|c|}
\hline & \multicolumn{2}{|l|}{$\mathrm{LTA}_{\mathrm{AA}}$} & $\begin{array}{l}\mathrm{p} \\
\text { value }\end{array}$ & \multicolumn{2}{|l|}{$\mathrm{TEG}_{\mathrm{AA}}$} & $\begin{array}{l}\mathrm{p} \\
\text { value }\end{array}$ & \multicolumn{2}{|c|}{$\mathrm{LTA}_{\mathrm{AA}}+\mathrm{TEG}_{\mathrm{AA}}$} & $\begin{array}{l}\mathrm{p} \\
\text { value }\end{array}$ \\
\hline Events, n (\%) & $8(13.6)$ & $37(9.9)$ & 0.399 & $13(12.7)$ & $32(9.7)$ & 0.384 & $9(23.7)$ & $36(9.2)$ & 0.025 \\
\hline $\mathrm{MI}, \mathrm{n}$ & 3 & 7 & 0.145 & 3 & 7 & 0.434 & 2 & 8 & 0.218 \\
\hline Hospitalization for UA, $n$ & 2 & 7 & 0.355 & 2 & 7 & 0.662 & 2 & 7 & 0.184 \\
\hline
\end{tabular}

$\mathrm{MI}=$ Myocardial infarction; $\mathrm{UA}=$ unstable angina; $\mathrm{TIA}=$ transient ischemic attack; $\mathrm{AR}=$ aspirin resistance; non- $\mathrm{AR}=$ non-aspirin resistance.

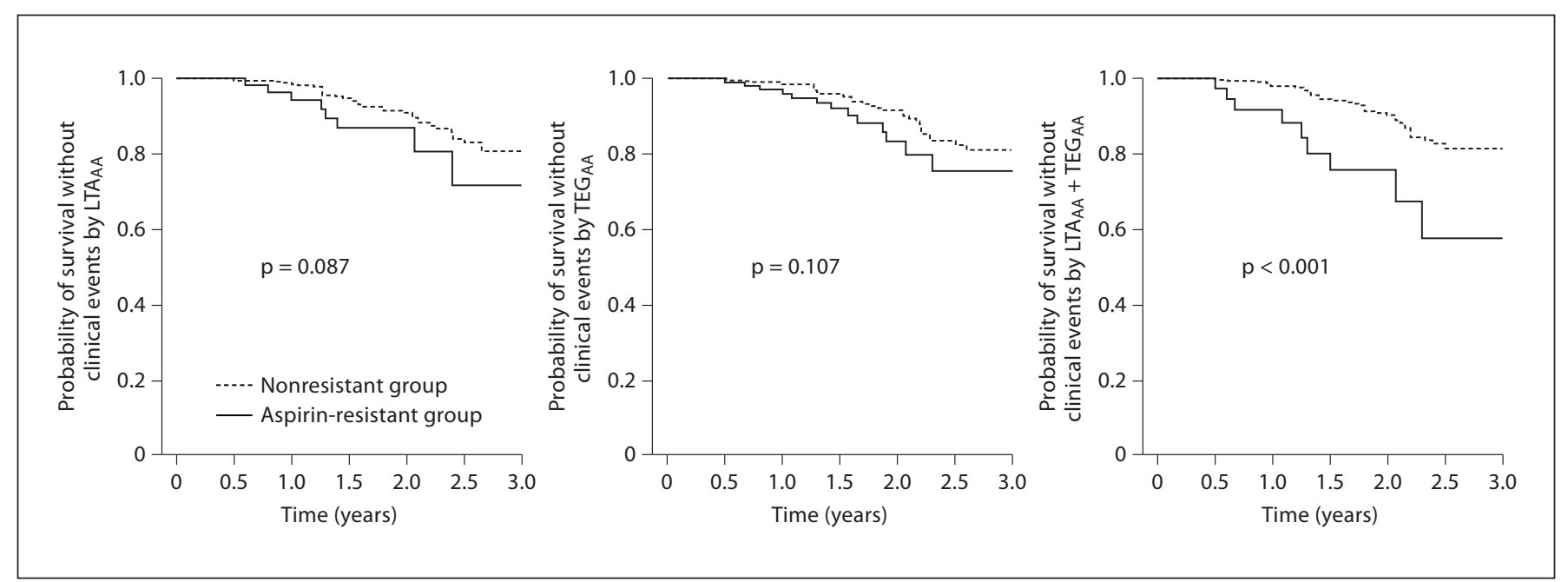

Fig. 1. Kaplan-Meier curves of the probability of freedom from composite endpoints according to response to aspirin therapy. Composite endpoints included myocardial infarction, stroke, transient ischemic attack, and unstable angina. Patients were defined as aspirin resistant by different methods.

that a history of cerebrovascular disease was an independent risk factor for clinical events. These results suggest that we should not ignore patients with a history of cerebrovascular disease.

Several studies have shown an association between diabetes and aspirin resistance in humans and in animal models [31, 32]. In most of these studies, an association between aspirin resistance and elevated $\mathrm{HbAlc}$ and increased fasting serum glucose levels was reported in diabetic patients [33-35]. The present study also found that fasting serum glucose level was a risk factor for aspirin resistance. Hyperglycemia may interfere with the acetylation process of platelets by aspirin, which may contribute to aspirin resistance in the presence of diabetes [13]. These studies suggest that glycemic control in CVD patients complicated by diabetes may improve the antithrombotic effects of aspirin.

CD62P is used as a marker of platelet activation. Dropinski et al. [36] revealed that P-selectin (CD62P) expression was increased in aspirin-treated aspirin-resistant patients with coronary artery disease compared with aspirin-sensitive patients or aspirin-treated healthy subjects. Andersen et al. [25] showed that the levels of soluble P-selectin (CD62P) were significantly higher in aspirin-resistant patients as compared to nonresistant patients in a group of 129 patients with acute myocardial 
Table 4. Comparison of the SNP allele changes in the COX-1 gene by different diagnostic criteria

\begin{tabular}{lll}
$\mathrm{LTA}_{\mathrm{AA}}$ & \\
\hline AR vs. non-AR & \\
\hline case & $\begin{array}{l}\text { control } \\
(\mathrm{n}=59)\end{array} \quad \begin{array}{l}\mathrm{p} \text { value } \\
(\mathrm{n}=372)\end{array}$ & OR $(95 \% \mathrm{CI})$
\end{tabular}

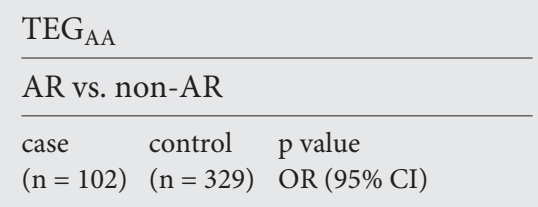

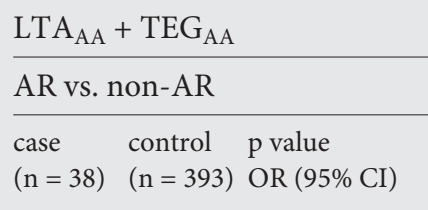

\section{rs1888943}

Allele

0.49

0.33

\begin{tabular}{lrr}
$\mathrm{C}$ & 118 & 741 \\
$\mathrm{~T}$ & 0 & \\
\hline
\end{tabular}

\section{rs1330344}

Allele

\begin{tabular}{lll} 
A & 61 & 451 \\
$\mathrm{G}$ & 57 & 291 \\
\hline rs3842787 & & \\
Allele &
\end{tabular}

204

655

3

$0 \quad 3$

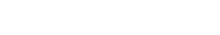

0.06

\begin{tabular}{lrr} 
Allele & & \\
$\mathrm{C}$ & 118 & 740 \\
$\mathrm{~T}$ & 0 & 2 \\
\hline
\end{tabular}

0.06

rs5787

Allele

$\begin{array}{rrr}0.69(0.47-1.02) & 110 & 402 \\ 1.45(0.95-2.14) & 94 & 254\end{array}$

0.06

$0.74(0.54-1.02) \quad 35 \quad 477$

0.01

0.57

0.43

$$
0.57
$$

$.35(0.99-1.86)$

$41 \quad 307$

$0.55(0.34-0.88)$

0.43 $1.82(1.13-2.92)$

\begin{tabular}{lrr}
$\mathrm{G}$ & 118 & 737 \\
$\mathrm{~A}$ & 0 & \\
\hline
\end{tabular}

\section{rs5789}

Allele

C

A

Allele

$204 \quad 654$

654
2

0.66

$\begin{array}{rr}76 & 782 \\ 0 & 2\end{array}$

0.37

0.21

0.49

\begin{tabular}{lllllll} 
G & 118 & 742 & 204 & 656 & 76 & 784 \\
\hline
\end{tabular}

$\mathrm{AR}=$ Aspirin resistance; non- $\mathrm{AR}=$ non-aspirin resistance

infarction. The present study agreed with these findings. These results imply that aspirin resistance may be associated with increased platelet reactivity.

Holmes et al. [37] showed that chronic therapy with either isosorbide $5^{\prime}$ mono-nitrate or transdermal nitroglycerine is associated with the development of vascular tolerance. Despite the induction of vascular tolerance, platelet responsiveness to nitroglycerine and sodium nitroprusside remains unaffected [37]. The present study demonstrated that administration of nitrates was a protective factor for aspirin resistance in elderly patients. This may be associated with increased production of exogenous nitric oxide, which can inhibit platelet aggregation. These findings suggest that nitrate therapy may improve the antiplatelet effects of aspirin.

Recently, Krasopoulos et al. [7] evaluated 2,930 patients with CVD receiving aspirin therapy for secondary prevention from 20 studies. The frequency of cardiovascular-related events including death, acute coronary syn- dromes, new cerebrovascular episodes, or failure of vascular intervention was higher in aspirin-resistant patients than in aspirin-sensitive patients ( 39 vs. $16 \%$; OR $=3.85$ ), as measured by various laboratory methods [7]. Snoep et al. [8] evaluated the rate of recurrence of cardiovascular events in 2,296 patients from 16 studies. The pooled OR for clinical ischemic events and the resulting OR for laboratory aspirin resistance was 4.37 (95\% CI 2.19-8.73), as assessed by different methods. When studies with all clinical events were pooled, the pooled OR was 3.78 (95\% CI 2.34-6.11) in aspirin-resistant patients [8]. Two metaanalyses have associated the occurrence of adverse clinical events with laboratory aspirin resistance defined by the PFA-100. A systematic review of 53 studies with 6,450 patients showed that aspirin-resistant patients were more likely to have vascular events than were nonresistant patients (relative risk 1.63; 95\% CI 1.16-2.28) [9]. Similarly, in another meta-analysis of 8 studies $(n=1,227)$, the global OR was 2.1 (95\% CI 1.4-3.4) for the recurrence of an 
ischemic event in aspirin-resistant patients, as evaluated by the PFA-100 [10]. The present study also found that aspirin-resistant patients had a significantly increased risk of cardiovascular events compared with nonresistant patients by $\mathrm{LTA}_{\mathrm{AA}}+\mathrm{TEG}_{\mathrm{AA}}$. These results suggest that laboratory aspirin resistance may be associated with the occurrence of adverse clinical outcomes.

Many studies focus on pharmacogenetic factors related to aspirin resistance. Genetic variants of COX-1, COX-2, platelet glycoproteins GPIa, GPIba, and GPIIIba, coagulation factor XIII, and TXA2 receptor have been reported in patients with aspirin resistance [38-40]. However, the data are inconsistent regarding $\mathrm{C} 50 \mathrm{~T}$ polymorphisms of COX-1 rs3842787, which may be because of race, inadequate assessment of phenotype and risk factors, or lack of a gold standard method for diagnosis. A much larger number of subjects is needed to clarify the association between aspirin resistance and pharmacogenetics.

COX-1 is a target enzyme of aspirin. COX-1 gene polymorphisms may affect the action of the enzyme, as well as its interaction with aspirin [41]. Aspirin resistance detected by COX-1-specific tests should be completely consistent with the aspirin resistance phenotype, as well as with COX-1 genetic variants, according to the primary principle that phenotype is determined by genotype. In addition, the gene difference in COX-1 associated with aspirin resistance significantly varies with racial status. Data from a review by Agúndez et al. [42] suggested that the major COX-1 targets in aspirin resistance are rs3842787 and rs5789 for European subjects, and rs3842789 and rs3842792 for African subjects. The present study showed that COX-1 rs1330344 is the target SNP for the Chinese Han population. rs1888943, rs3842787, rs5787, rs5789, and rs5794 are rare in Han. The promoter $-1676 \mathrm{G}$ allele appears to contribute significantly to aspirin resistance in the Chinese Han population. rs1330344 has a high frequency in the Chinese population. The frequency of minor allele $-1676 \mathrm{G}$ is 0.40 . In the Japanese population, rs1330344 is also highly prevalent, and the $-1676 \mathrm{C}$ allele has a protective effect against functional dyspepsia in Japanese women; $-1676 \mathrm{~A}$ is a risk factor for aspirin-induced peptic ulcer in Japanese [43, 44]. Moreover, the gene differences for COX-1 are highly consistent between the Chinese and Japanese populations. Thus, rs1330344 may be a common tag SNP in the COX-1 gene for Asian patients with aspirin resistance. We should pay more attention to elderly Chinese patients with gene differences for COX-1 and evaluate the genetic polymorphism of COX-1 in aspirin-resistant patients. Establishing the relationship between aspirin resistance and ge- netic polymorphism of COX-1 may provide more effective and less hazardous treatment strategies in patients with aspirin resistance.

There are several possible therapeutic options of intervention in genetic polymorphism of COX-1 for aspirin resistance. Firstly, increasing the dose of aspirin may possibly reduce the rate of the poor response to aspirin in individual patients [45]. Secondly, aspirin may be switched to clopidogrel, prasugrel, or cilostazol, which have been found to result in decreased rates of clinical events in patients with CVD [46-48]. Thirdly, adding clopidogrel or other antiplatelet drugs to aspirin is another approach. Further studies are needed to focus on the relationship between aspirin dose and genetic polymorphism of the COX-1.

The present study had several limitations. Firstly, the frequency of aspirin resistance in elderly male patients with CVD was valid for the dose of 75-100 mg/day of aspirin, but we did not investigate the other suggested doses of $162 \mathrm{mg} /$ day and $325 \mathrm{mg} /$ day. Secondly, the study was conducted in the Chinese Han population, and the relevance and applicability of these results to other populations is unknown. Thirdly, the relatively low number of subjects may have reduced the ability of this study to detect and relate rarer gene variants with aspirin resistance. Fourthly, there is no universally agreed upon way to assess aspirin resistance, and this study relied on 3 different measures.

In conclusion, we found that the frequency of aspirin resistance in elderly Chinese patients with CVD is considerably higher in patients with cerebrovascular disease, higher serum levels of fasting serum glucose and CD62P, and changes in the medication taken. Aspirin resistance, evaluated by $\mathrm{LTA}_{\mathrm{AA}}+\mathrm{TEG}_{\mathrm{AA}}$, is associated with an increased risk of clinical events in elderly Chinese patients with CVD. The variant G-allele of COX-1 rs1330344 is significantly associated with aspirin resistance defined by $\mathrm{LTA}_{\mathrm{AA}}+\mathrm{TEG}_{\mathrm{AA}}$ in the present study. These findings may provide important information for individualized aspirin therapy.

\section{Acknowledgment}

This work was supported by the Healthcare Fund of China (07BJZ01) and the Ministry of Science and Technology of China (2009BAI86B04).

\section{Disclosure Statement}

The authors have no conflicts of interest with this paper. 


\section{References}

$>1$ Yazdanyar A, Newman AB: The burden of $>14$ Schafer AI: Genetic and acquired determicardiovascular disease in the elderly: morbidity, mortality, and costs. Clin Geriatr Med 2009;25:563-577.

2 Zheng Y SR, Kwan T, Yu C, Kwan J, Chen SL, $\mathrm{Hu}$ D: Evolving cardiovascular disease prevalence, mortality, risk factors, and the metabolic syndrome in China. Clin Cardiol 2009; 32:491-497.

-3 Collaboration AT: Collaborative meta-analysis of randomised trials of antiplatelet therapy for prevention of death, myocardial infarction, and stroke in high risk patients. BMJ 2002;324:71-86.

4 Gum PA, Kottke-Marchant K, Welsh PA, White J, Topol EJ: A prospective, blinded determination of the natural history of aspirin resistance among stable patients with cardiovascular disease. J Am Coll Cardiol 2003; 41:961-965.

$>5$ Chen WH, Cheng X, Lee PY, Ng W, Kwok JY, Tse HF, Lau CP: Aspirin resistance and adverse clinical events in patients with coronary artery disease. Am J Med 2007;120: 631-635.

6 Feher G, Feher A, Pusch G, Koltai K, Tibold A, Gasztonyi B, Papp E, Szapary L, Kesmarky G, Toth K: Clinical importance of aspirin and clopidogrel resistance. World J Cardiol 2010;2:171-186.

7 Krasopoulos G, Brister SJ, Beattie WS, Buchanan MR: Aspirin 'resistance' and risk of cardiovascular morbidity: systematic review and meta-analysis. BMJ 2008;336:195-198.

$>8$ Snoep JD, Hovens MM, Eikenboom JC, van der Bom JG, Huisman MV: Association of laboratory-defined aspirin resistance with a higher risk of recurrent cardiovascular events: a systematic review and meta-analysis. Arch Intern Med 2007;167:1593-1599.

-9 Crescente M, Di Castelnuovo A, Iacoviello L, Vermylen J, Cerletti C, de Gaetano G: Response variability to aspirin as assessed by the platelet function analyzer (PFA)-100: a systematic review. Thromb Haemost 2008; 99:14-26.

-10 Reny JL, De Moerloose P, Dauzat M, Fontana P: Use of the PFA-100 closure time to predict cardiovascular events in aspirin-treated cardiovascular patients: a systematic review and meta-analysis. J Thromb Haemost 2008;6: 444-450.

11 Kasotakis G, Pipinos II, Lynch TG: Current evidence and clinical implications of aspirin resistance. J Vasc Surg 2009;50:1500-1510.

-12 Feher G, Feher A, Pusch G, Koltai K, Tibold A, Gasztonyi B, Papp E, Szapary L, Kesmarky G, Toth K: Clinical importance of aspirin and clopidogrel resistance. World J Cardiol 2010;2:171-186.

13 Ajjan R, Storey RF, Grant PJ: Aspirin resistance and diabetes mellitus. Diabetologia 2008;51:385-390. nants of individual variability of response to antiplatelet drugs. Circulation 2003;108: 910-911.

15 Tantry US, Mahla E, Gurbel PA: Aspirin resistance. Prog Cardiovasc Dis 2009;52:141152.

16 Cao J, Liu L, Fan L, Chen T, Hu G, Hu Y, Zhu B, Li J, Wang H, Li X: The prevalence, risk factors and prognosis of aspirin resistance in elderly male patients with cardiovascular disease. Aging Male 2012;15:140-147.

17 Tantry US BK, Gurbel PA: Overestimation of platelet aspirin resistance detection by thromboleastograph platelet mapping and validation by conventional aggreagometry using arachidonic acid stimulation. J Am Coll Cardiol 2005;1705-1709.

18 Lordkipanidze M, Pharand C, Schampaert E, Turgeon J, Palisaitis DA, Diodati JG: A comparison of six major platelet function tests to determine the prevalence of aspirin resistance in patients with stable coronary artery disease. Eur Heart J 2007;28:17021708.

19 Alpert JS, Thygesen K, Antman E, Bassand JP: Myocardial infarction redefined - a consensus document of the Joint European Society of Cardiology/American College of Cardiology Committee for the redefinition of myocardial infarction. J Am Coll Cardiol 2000;36:959-969.

20 Anderson JL, Adams CD, Antman EM, Bridges CR, Califf RM, Casey DE Jr, Chavey WE 2nd, Fesmire FM, Hochman JS, Levin TN, Lincoff AM, Peterson ED, Theroux P, Wenger NK, Wright RS, Smith SC Jr, Jacobs AK, Halperin JL, Hunt SA, Krumholz HM, Kushner FG, Lytle BW, Nishimura R, Ornato JP, Page RL, Riegel B: ACC/AHA 2007 guidelines for the management of patients with unstable angina/non ST-elevation myocardial infarction: a report of the American College of Cardiology/American Heart Association Task Force on Practice Guidelines (Writing Committee to Revise the 2002 Guidelines for the Management of Patients With Unstable Angina/Non ST-Elevation Myocardial Infarction) - developed in collaboration with the American College of Emergency Physicians, the Society for Cardiovascular Angiography and Interventions, and the Society of Thoracic Surgeons: endorsed by the American Association of Cardiovascular and Pulmonary Rehabilitation and the Society for Academic Emergency Medicine. Circulation 2007;116:e148-e304.

21 Gurbel PA, Bilden KP, Di Chiara J, Newcomer J, Weng J, Neerchal W, Gesheff NK, et al: Evaluation of dose-related effects of aspirin on platelet function: results from the Aspirin-Induced Platelet Effect (ASPECT) study. Circulation 2007;115:3156-3164.
22 Tantry US, Bliden KP, Gurbel PA: Overestimation of platelet aspirin resistance detection by thrombelastograph platelet mapping and validation by conventional aggregometry using arachidonic acid stimulation. J Am Coll Cardiol 2005;46:1705-1709.

23 Carroll RC, Worthington RE, Craft RM, Snider CC, Dakin PA, Wortham DC, Scott J, Jarrett A: Post interventional cardiology urinary thromboxane correlates with PlateletMapping detected aspirin resistance. Thromb Res 2010;125:e118-e122.

24 Gum PA, Kottke-Marchant K, Poggio ED, Gurm H, Welsh PA, Brooks L, Sapp SK, Topol EJ: Profile and prevalence of aspirin resistance in patients with cardiovascular disease. Am J Cardiol 2001;88:230-235.

-25 Andersen K, Hurlen M, Arnesen H, Seljeflot I: Aspirin non-responsiveness as measured by PFA-100 in patients with coronary artery disease. Thromb Res 2002;108:37-42.

26 Ozben S, Ozben B, Tanrikulu AM, Ozer F, Ozben T: Aspirin resistance in patients with acute ischemic stroke. J Neurol 2011;258: 1879-1986.

27 Nidhinandana S, Changchit S: Prevalence of aspirin resistance in stroke patients in Phramongkutklao Hospital. J Med Assoc Thai 2011;93(suppl 6):S51-S54.

$>28$ Lee JH, Cha JK, Lee SJ, Ha SW, Kwon SU: Addition of cilostazol reduces biological aspirin resistance in aspirin users with ischaemic stroke: a double-blind randomized clinical trial. Eur J Neurol 2009; 17:434-442.

-29 Englyst NA, Horsfield G, Kwan J, Byrne CD: Aspirin resistance is more common in lacunar strokes than embolic strokes and is related to stroke severity. J Cereb Blood Flow Metab 2008;28:1196-1203.

30 Grotemeyer KH, Scharafinski HW, Husstedt IW: Two-year follow-up of aspirin responder and aspirin non responder: a pilot-study including 180 post-stroke patients. Thromb Res 1993;71:397-403.

31 Watala C, Ulicna O, Golanski J, Nocun M, Waczulikova I, Markuszewski L, Drzewoski J: High glucose contributes to aspirin insensitivity in streptozotocin-diabetic rats: a multiparametric aggregation study. Blood Coagul Fibrinolysis 2006;17:113-124.

32 Watala C, Golanski J, Pluta J, Boncler M, Rozalski M, Luzak B, Kropiwnicka A, Drzewoski J: Reduced sensitivity of platelets from type 2 diabetic patients to acetylsalicylic acid (aspirin) - its relation to metabolic control. Thromb Res 2004;113:101-113.

33 Pulcinelli FM, Riondino S: More on aspirin resistance: position paper of the Working Group on Aspirin Resistance - proposal for a laboratory test guiding algorithm. J Thromb Haemost 2006;4:485-487. 
34 Ertugrul DT, Tutal E, Yildiz M, Akin O, Yalcin AA, Ure OS, Yilmaz H, Yavuz B, Deveci OS, Ata N, Kucukazman M: Aspirin resistance is associated with glycemic control, the dose of aspirin, and obesity in type 2 diabetes mellitus. J Clin Endocrinol Metab 2010;95: 2897-901.

35 Cohen HW, Crandall JP, Hailpern SM, Billett $\mathrm{HH}$ : Aspirin resistance associated with $\mathrm{HbAlc}$ and obesity in diabetic patients. J Diabetes Complications 2008;22:224-228.

-36 Dropinski J, Jakiela B, Sanak M, Wegrzyn W, Biernat M, Dziedzina S, Plutecka H, Szczeklik A: The additive antiplatelet action of clopidogrel in patients with coronary artery disease treated with aspirin. Thromb Haemost 2007;98:201-209.

-37 Holmes AS, Chirkov YY, Willoughby SR, Poropat S, Pereira J, Horowitz JD: Preservation of platelet responsiveness to nitroglycerine despite development of vascular nitrate tolerance. Br J Clin Pharmacol 2005;60:355363.

- 38 Tassies D: Pharmacogenetics of antithrombotic drugs. Curr Pharm Des 2006;12:24252435.

39 Oh SH, Kim YH, Park SM, Cho SH, Park JS, Jang AS, Park SW, Uh ST, Lee YM, Kim MK, Choi IS, Hong CS, Lee YW, Lee JY, Choi BW, Park BL, Shin HD, Park CS: Association analysis of thromboxane A synthase 1 gene polymorphisms with aspirin intolerance in asthmatic patients. Pharmacogenomics 2011;12:351-363.
40 Lev EI, Patel RT, Guthikonda S, Lopez D, Bray PF, Kleiman NS: Genetic polymorphisms of the platelet receptors P2Y(12), P2Y(1) and GP IIIa and response to aspirin and clopidogrel. Thromb Res 2007;119:355360.

41 Goodman T, Sharma P, Ferro A: The genetics of aspirin resistance. Int J Clin Pract 2007;61: 826-834.

42 Agúndez JA, Martinez C, Perez-Sala D, Carballo M, Torres MJ, Garcia-Martin E: Pharmacogenomics in aspirin intolerance. Curr Drug Metab 2009;10:998-1008.

43 Arisawa T, Tahara T, Shibata T, Nagasaka M, Nakamura M, Kamiya Y, Fujita H, Yoshioka D, Arima Y, Okubo M, Hirata I, Nakano $\mathrm{H}$ : Association between genetic polymorphisms in the cyclooxygenase-1 gene promoter and peptic ulcers in Japan. Int J Mol Med 2007;20:373-378.

-44 Arisawa T, Tahara T, Shibata T, Nagasaka M, Nakamura M, Kamiya Y, Fujita H, Yoshioka D, Arima Y, Okubo M, Hirata I, Nakano H: Genetic polymorphisms of cyclooxygenase-1 (COX-1) are associated with functional dyspepsia in Japanese women. J Womens Health (Larchmt) 2008;17:1039-1043.
5 Gurbel PA, Bilden KP, DiChiara J, Newcomer J, Weng W, Neerchal NK, Gesheff T, Chaganti SK, Etherington A, Tantry US: Evaluation of dose-related effects of aspirin on platelet function: results from the AspirinInduced Platelet Effect (ASPECT) study. Circulation 2007;115:3156-3164.

46 Yusuf S, Zhao F, Mehta SR, Chrolavicius S, Tognoni G, Fox KK: Effects of clopidogrel in addition to aspirin in patients with acute coronary syndromes without ST-segment elevation. N Engl J Med 2001;345:494-502.

-47 Zhang Z, Foster JK, Kolm P, Jurkovitz CT, Parker KM, Murrah NV, Anderson GT, Douglas JS Jr, Weintraub WS: Reduced 6-month resource use and costs associated with cilostazol in patients after successful coronary stent implantation: results from the Cilostazol for RESTenosis (CREST) trial. Am Heart J 2006;152:770-776.

48 Mahoney EM, Wang K, Arnold SV, Proskorovsky I, Wiviott $S$, Antman E, Braunwald E, Cohen DJ: Cost-effectiveness of prasugrel versus clopidogrel in patients with acute coronary syndromes and planned percutaneous coronary intervention: results from the trial to assess improvement in therapeutic outcomes by optimizing platelet inhibition with prasugrel-thrombolysis in myocardial infarction TRITON-TIMI 38. Circulation 2010; 121:71-79. 